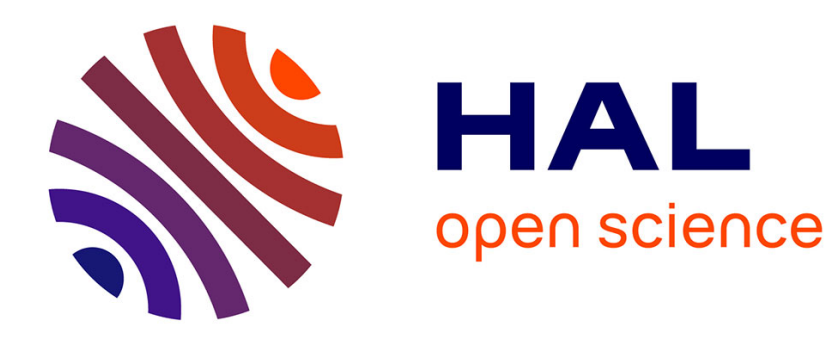

\title{
Discussions on observability and invertibility
}

Wei Kang, Jean-Pierre Barbot

\section{To cite this version:}

Wei Kang, Jean-Pierre Barbot. Discussions on observability and invertibility. NOLCOS, IFAC, Aug 2007, Pretoria, South Africa. inria-00182433

\section{HAL Id: inria-00182433 \\ https://hal.inria.fr/inria-00182433}

Submitted on 25 Oct 2007

HAL is a multi-disciplinary open access archive for the deposit and dissemination of scientific research documents, whether they are published or not. The documents may come from teaching and research institutions in France or abroad, or from public or private research centers.
L'archive ouverte pluridisciplinaire HAL, est destinée au dépôt et à la diffusion de documents scientifiques de niveau recherche, publiés ou non, émanant des établissements d'enseignement et de recherche français ou étrangers, des laboratoires publics ou privés. 


\title{
Discussions on observability and invertibility
}

\author{
W. Kang and J-P Barbot*
}

October 26, 2007

\begin{abstract}
In this paper, new definitions of observability are introduced. They include states and/or inputs estimation, as well as partial or global observability. From these definitions, problems of left invertibility, fault detection, observability, identifiability, unidirectional synchronization,... can be studied in the same context. Moreover, we propose the definitions for both nonlinear continuous-time systems and switched systems. Two examples are introduced to illustrate the efficiency of the proposed definitions and related propositions introduce in the paper.
\end{abstract}

\section{Introduction}

In this paper, the concept of observability for nonlinear systems and switched systems is investigated with new definitions in an extended context. Relative to the classical concept of observability, the proposed definitions are applicable to a much broader family of variables including unmeasured state, unknown input, unknown parameters, and even uncertainties and disturbances in a control system. Moreover, the proposed definition takes into account the notion of partial observability which may be useful, for example, in the context of networked systems. In addition, the first definition in this paper has a distinctive extension to switched systems and thus taking into account also the partial observability with respect to the time (i.e. some variable may be unobservable during some bounded time interval). It is well known that the definition of linear observability is universal for all linear systems, but there exist many different definitions of observability in the literature of nonlinear control systems. Without exhaustibility purposes, these

${ }^{*}$ W. Kang is with Department of Applied Mathematics, Naval Postgraduate School, Monterey, CA and J.P. Barbot (Member of Project ALIEN-INRIA-Futurs) is with ECS, ENSEA, 6 avenue du Ponceau, 95014 Cergy, FRANCE 
definitions include weakly local observability (Herman \& al., 1977), algebraic observability (Diop \& al., 1991), infinitesimal observability (Gauthier \& al., 2001), unboundedness observability (Angeli \& al., 1999),... It is a technical question at the crossway of several factors such as the generality of the concept, easy to check, and practical observer design.

In this paper, the notation $Z$ represents a variable or function associated with a control system. The first definition of observability proposed in this paper is the Z-observability. It is an effort to take into account the consideration of three factors that are not considered all together in existing definitions:

- Partial observability (observability of a part of the system variables, not all the state variables)

- Observability with partial model of a dynamical system, i.e. part of the equations in the system model is completely unknown (in contrast to the case with full known model with possibly unknown parameters);

- The observability of unknown input and unknown parameters.

This new definition is inspired by the observability definition adopted by moving horizon type of observers. However, the following definition has some fundamental differences from the existing ones. It defines the observability of individual variables, instead of the entire system. In addition to the classical case of observability of the entire system, this definition is applicable to the estimation of a subset of variables. It is also applicable to the unknown input and unknown parameters in a system. Consequently, this definition can be easily applied to fault detection and, more particularly, to some approaches using subsystems of connected or switched systems. Such definition can also be used in many observer based applications, such as cryptography of chaotic systems; identification problems; and general left invertible problems.

After the introduction of $Z$-observability, it is introduced the notion of $Z\left(T_{N}\right)$-observability for switched systems. This new definition takes into consideration of five factors not considered all together in classical definitions:

- Partial state observability

- Observability with partial model of a dynamical system

- The observability of unknown input and unknown parameters 
- Systems with algebraic constraints

- Partial time observability

One of the main advantage of this is to relax the assumption that requires all switched subsystems be observable (see for example (Bemporad \& al., 2000; Boutat \& al., 2004; Vu \& al., 2006)). The efficiency of both definitions $Z$-observability and $Z\left(T_{N}\right)$-observability are highlighted with two examples: the first one is dedicated to Fault Detection in networked oscillator and the second one is dedicated to an academical switched system.

\section{$2 \quad Z$-observability for smooth system}

Consider a general nonlinear control system

$$
\begin{aligned}
\dot{\xi} & =f(t, \xi, u), \quad \xi \in \mathbb{R}^{n}, \quad u \in \mathbb{R}^{m} \\
Y & =h(t, \xi, u)
\end{aligned}
$$

Suppose $z=Z(t, x, u)$ is a variable to be estimated. In the following, $U$ represents an open and connected set in the time-state-control space $\mathbb{R} \times \mathbb{R}^{n} \times \mathbb{R}^{m}$. For the input in any time interval $\left[t_{0}, t_{1}\right]$, we assume that $u(t)$ is bounded and $C^{\infty}$ at all but finite many points in $\left[t_{0}, t_{1}\right]$. At each discontinuous point $\left.t_{i} \in\right] t_{0}, t_{1}\left[, \lim _{t \rightarrow t_{i}^{+}} u(t)\right.$ and $\lim _{t \rightarrow t_{i}^{-}} u(t)$ exist. For the state, we assume $\xi(t)$ absolutely continuous. If $(\xi(t), u(t))$ satisfies the differential equation in (2.1) for all $t$ in $\left[t_{0}, t_{1}\right]$ except for finite many points, then $(t, \xi(t), u(t))$ is called a trajectory. In this note, equations involving $u(t)$ always mean "equal almost everywhere," and the notation is "a.e. in $\left[t_{0}, t_{1}\right] . "$

Definition 1 The function $z=Z(t, x, u)$ is said to be $Z$-observable in $U$ with respect to the system 2.1 if for any two trajectories, $\left(t, \xi^{i}(t), u^{i}(t)\right)$, $i=1,2$, in $U$ defined on a same interval $\left[t_{0}, t_{1}\right]$, the equality

$$
h\left(\xi^{1}(t), u^{1}(t)\right)=h\left(\xi^{2}(t), u^{2}(t)\right), \quad \text { a.e. in }\left[t_{0}, t_{1}\right]
$$

implies

$$
Z\left(t, \xi^{1}(t), u^{1}(t)\right)=Z\left(t, \xi^{2}(t), u^{2}(t)\right), \text { a.e. in }\left[t_{0}, t_{1}\right]
$$

Now, suppose for any trajectory $(t, \xi(t), u(t))$ in $U$, there always exists an open set $U_{1} \subset U$ so that $Z(t, x, u)$ is $Z$-observable in $U_{1}$. Then, $z=Z(t, x, u)$ is said to be locally $Z$-observable in $U$. 
For simplicity, " $Z$-observable in $U$ " means " $Z$-observable in $U$ with respect to the system 2.1". In linear control theory, the control input is assumed to be a known variable. So, $Y=[y, u]^{T}$ where $y$ is the output in the classical definition. Then, the observability of a linear control system is equivalent to the observability of all state variables in $x$. For nonlinear systems, all the state variables in an uniformly observable system must be observable under the definition 1 with the function $Z(t, x, u)$ equal to $x$ and $Y=[y, u]^{T}$. However, a system that is not uniformly observable may be observable under the definition 1 with particular $Z(t, x, u)$. Thus, a system could be unobservable under all existing definitions, but part of the state variables can be observable. This partial observability is a new concept that is especially important for subsystems in a system of systems, in which the observability of the entire system is either impossible or unnecessary.

It is also important to notice that the input $u$ is a variable treated equally as the state variable $x$ in the definition 1 . So, it automatically handles both the left invertibility problem and the parameter identification problem. Moreover, it will be proved later that $z$ is $Z$-observable implies $\dot{z}$ is also $Z$-observable (when $\dot{z}$ exists), no matter we know or don't know the dynamic equations in the model that are not directly related to $\dot{z}$.

The local $Z$-observability is defined differently from some typical local properties. In fact, the meaning of local in Definition 1 is not around a point in the state space. The local neighborhood $U_{1}$ is around a trajectory. A local observer around a single point would be too restrictive for many applications. Meanwhile, it is possible to design nonlinear observers applicable to non-local trajectories, but with local initial estimation (the observer converges if the initial estimation error is small), like the observer in (Krener \& al., 2003).

If a variable is observable, it can be estimated by Kalman type of filters (EKF or UKF), or moving horizon type of observables (Kang, 2006) and (Gong \& al., 2007), sliding mode observer (Levant, 1998), high gain observer (Gauthier \& al., 2001),... but the choice and design of observers are beyond the scoop of this paper.

In the next, we prove several sufficient conditions for the $Z$-observability. Some of them are numerically checkable. For the simplicity of notations, we denote $\xi_{0}=\xi, \xi_{1}=u$. We define the differentiation operator $D$ by

$$
\begin{aligned}
D\left(\xi_{j}\right) & =\xi_{j+1}, \quad j \geq 1 \\
D\left(K\left(t, \xi_{0}, \xi_{1}, \cdots, \xi_{j}\right)\right) & =\frac{\partial K}{\partial t}+\frac{\partial \bar{K}}{\partial \xi_{0}} f\left(\xi_{0}, \xi_{1}\right) \\
& +\frac{\partial K}{\partial\left(\xi_{1}, \ldots, \xi_{j}\right)}\left[\xi_{2}, \ldots, \xi_{j+1}\right]^{T}
\end{aligned}
$$


Lemma 1 Suppose there exists a function $g(\cdot)$ so that

$$
z=Z(t, \xi, u)=g\left(t, h, D h, \cdots, D^{k-1} h\right)
$$

for all $(t, \xi, u) \in U$ (the equality implies that the value of $\xi_{j+1}$ can be arbitrary for $j \geq 1)$. Then $z(t, x, u)$ is $Z$ observable.

Proof. Given two trajectories $\left(\xi^{1}(t), u^{1}(t)\right)$ and $\left(\xi^{2}(t), u^{2}(t)\right)$ in $U$ with outputs $Y^{1}(t)$ and $Y^{2}(t)$. Suppose

$$
Y^{1}(t)=Y^{2}(t), \quad t \in\left[t_{0}, t_{1}\right]
$$

It is obvious that for $i=1,2$

$$
\begin{aligned}
& \dot{Y}^{i}(t)=\left.D\left(h\left(t, \xi^{i}, u^{i}\right)\right)\right|_{\xi_{0}=\xi^{i}(t), \xi_{1}=u^{i}(t), \xi_{2}=u^{i}(t)} \\
& \frac{d^{j}}{d t^{j}}\left(Y^{i}(t)\right)=\left.D^{j}\left(h\left(t, \xi^{i}, u^{i}\right)\right)\right|_{\xi_{0}=\xi^{i}(t), \cdots, \xi_{j+1}=\left(u^{i}\right)^{(j)}(t)}
\end{aligned}
$$

Because of $(2.2)$, we know $\frac{d^{j}}{d t^{j}}\left(Y^{1}(t)\right)=\frac{d^{j}}{d t^{j}}\left(Y^{2}(t)\right)$ for $0 \leq j \leq k-1$. Therefore,

$$
\begin{aligned}
z\left(t, \xi^{1}(t), u^{1}(t)\right) & =\left.g\left(t, h, D h, . ., D^{k-1} h\right)\right|_{\xi^{1}(t), . .,\left(u^{1}\right)^{(k-1)}(t)} \\
& =g\left(t, Y^{1}(t), \dot{Y}^{1}(t), . ., \frac{d^{k-1}}{d t^{k-1}} Y^{1}(t)\right) \\
& =g\left(t, Y^{2}(t), \dot{Y}^{2}(t), . ., \frac{d^{k-1}}{d t^{k-1}} Y^{2}(t)\right) \\
& =z\left(t, \xi^{2}(t), u^{2}(t)\right)
\end{aligned}
$$

So, the variable $z$ is observable.

Lemma 2 Let $U \subset \mathbb{R} \times \mathbb{R}^{n} \times \mathbb{R}^{m}$ be an open set. Suppose $z \in \mathbb{R}$ is a scaler valued variable. Suppose there are $k$ functions:

$$
v=\left[\begin{array}{lll}
v_{1}\left(t, \xi_{0}, \xi_{1}\right) & \cdots & v_{k}\left(t, \xi_{0}, \xi_{1}\right)
\end{array}\right]^{T}
$$

from the row components of the vector

$$
\left(Y^{T}, D Y^{T}, \ldots, D^{l-1} Y^{T}\right)^{T}
$$

so that
(1) $\frac{\partial v}{\partial \xi_{j}}=0$, for $j \geq 2,\left(t, \xi_{0}, \xi_{1}\right) \in U$
(2) $\operatorname{rank}\left(\frac{\partial v}{\partial\left(\xi_{0}, \xi_{1}\right)}\right)=k$, for $\left(t, \xi_{0}, \xi_{1}\right) \in U$ 
(3) for all $\left(t, \xi_{0}, \xi_{1}\right) \in U$

$$
\frac{\partial}{\partial\left(\xi_{0}, \xi_{1}\right)} Z\left(t, \xi_{0}, \xi_{1}\right) \in \text { row space of } \frac{\partial v}{\partial\left(\xi_{0}, \xi_{1}\right)},
$$

Then $z=Z(t, \xi, u)$ is locally $Z$-observable in $U$.

Proof. Given any point $\left(t_{0}, \xi^{0}, u^{0}\right)$ in $U$. There exists an open ball $B\left(t_{0}, \xi^{0}, u^{0}\right)$ of $\left(t_{0}, \xi^{0}, u^{0}\right)$ in $\mathbb{R} \times \mathbb{R}^{n} \times \mathbb{R}^{m}$ and a mapping

$$
w(t, \xi, u): \mathbb{R} \times \mathbb{R}^{n} \times \mathbb{R}^{m} \rightarrow \mathbb{R}^{n+m-k}
$$

so that $\left(t, v(t, \xi, u)^{T}, w(t, \xi, u)^{T}\right)^{T}$ is a diffeomorphism on $B\left(t_{0}, \xi_{0}, u_{0}\right)$. Therefore,

$$
\frac{\partial z}{\partial w}=\frac{\partial z}{\partial(\xi, u)}\left[\begin{array}{l}
\frac{\partial \xi}{\partial w} \\
\frac{\partial u}{\partial w}
\end{array}\right]
$$

However, there exists a row vector $a(t) \in \mathbb{R}^{k}$ so that

$$
\frac{\partial z}{\partial(\xi, u)}=a(t) \frac{\partial v}{\partial(\xi, u)}
$$

we have

$$
\frac{\partial z}{\partial w}=a(t) \frac{\partial v}{\partial(\xi, u)}\left[\frac{\partial \xi}{\partial w} \frac{\partial u}{\partial w}\right]=a(t) \frac{\partial v}{\partial w}=0
$$

Therefore, $z=Z(t, \xi(t, v, w), u(t, v, w))$ is a function of $v$, which is independent of $w$. Because $v$ is from the vector (2.3), $z$ is a function of $Y, D Y, \cdots, D^{l-1} Y$. Therefore, $z=o(t, \xi, u)$ is $Z$-observable in $B\left(t_{0}, \xi_{0}, u_{0}\right)$.

Given any trajectory $(t, \xi(t), u(t))$ in $U$. Then it is contained in some compact subset of $U$. There exist finite many open balls

$$
B\left(s_{1}, \xi\left(s_{1}\right), u\left(s_{1}\right)\right), B\left(s_{2}, \xi\left(s_{2}\right), u\left(s_{2}\right)\right), \cdots, B\left(s_{p}, \xi\left(s_{p}\right), u\left(s_{p}\right)\right)
$$

so that the entire trajectory stays inside the open set

$$
U_{1}=\cup_{i=1}^{p} B\left(s_{i}, \xi_{i}, u_{i}\right)
$$

and $z$ is Z-observable in every ball. There exists an open subset $U_{2} \subset U_{1}$ containing the trajectory so that, for any two points $\left(t, \xi^{i}, u^{i}\right) \in U_{2}$ with the same $t$, both points must lie in a same ball $B\left(s_{i}, \xi\left(s_{i}\right), u\left(s_{i}\right)\right)$. In $U_{2}, z$ must be $Z$-observable because it is observable in all the balls $B\left(s_{i}, \xi\left(s_{i}\right), u\left(s_{i}\right)\right)$. So, $z$ is locally Z-observable in $U$. 
Lemma 3 Consider a control system

$$
\begin{aligned}
\dot{\xi} & =f(t, \xi, u) \\
Y & =h(t, \xi, u)
\end{aligned}
$$

Assume $z=Z(t, \xi, u)$ is $Z$-observable in $U$ satisfying

$$
\frac{\partial Z(t, \xi, u)}{\partial u}=0
$$

Then,

$$
D z=\frac{\partial Z(t, \xi, u)}{\partial t}+\frac{\partial Z(t, \xi, u)}{\partial \xi} f(t, \xi, u)
$$

is $Z$-observable in $U$.

Proof. Given two trajectories $\left(t, \xi^{1}(t), u^{1}(t)\right)$ and $\left(t, \xi^{2}(t), u^{2}(t)\right)$. Assume $h\left(\left(t, \xi^{1}(t), u^{1}(t)\right)-h\left(\left(t, \xi^{2}(t), u^{2}(t)\right)=0\right.\right.$ for all $t$.

Then, $Z\left(t, \xi^{1}(t), u^{1}(t)\right)=Z\left(t, \xi^{2}(t), u^{2}(t)\right)$ for all $t$. Therefore,

$$
\begin{aligned}
\left.D z\right|_{\xi=\xi^{1}(t), u=u^{2}(t)} & =\frac{d}{d t} Z\left(t, \xi^{1}(t), u^{1}(t)\right) \\
& =\frac{d}{d t} Z\left(t, \xi^{2}(t), u^{2}(t)\right) \\
& =\left.D z\right|_{\xi=\xi^{2}(t), u=u^{2}(t)}
\end{aligned}
$$

So, $D z$ is $Z$-observable.

It is also interesting to deduce the $Z$-observability of variables constrained by algebraic equations. It is a result applicable to systems defined by DAEs.

Lemma 4 Consider a sequence of functions

$$
z_{i}=Z_{i}(t, \xi, u), \quad i=1,2, \cdots, k
$$

defined in an open set $U$. Suppose $z_{i}$ is locally observable in $U$ for $i \geq k_{1}+1$. Suppose $g(\cdot)$ is a $C^{1}$ function defined in the range of $\left(z_{1}, \cdots, z_{k}\right)$ satisfying

$$
g\left(z_{1}, z_{2}, \cdots, z_{k}\right)=0
$$

along all trajectories and

$$
\frac{\partial g}{\partial\left(z_{1}, \cdots, z_{k_{1}}\right)} \neq 0
$$

Then, $z_{1}=Z_{1}(t, \xi, u), \cdots, z_{k_{1}}=Z_{k_{1}}(t, \xi, u)$ are locally $Z$-observable in $U$. 
Proof. Given any point $\left(t_{0}, \xi^{0}, u^{0}\right)$ in the closure of a trajectory $(t, \xi(t), u(t))$ (If $u(t)$ is continuous, no need to consider the closure). Consider the point

$$
\left(z_{10}, \cdots, z_{k 0}\right)=\left(Z_{1}\left(t_{0}, \xi^{0}, u^{0}\right), \cdots, Z_{k}\left(t_{0}, \xi^{0}, u^{0}\right)\right)
$$

in $\mathbb{R}^{k}$. Then there exists an open neighborhood $V_{1}$ around $\left(z_{10}, \cdots, z_{k_{1} 0}\right)$ in $\mathbb{R}^{k_{1}}$; and an open neighborhood $V_{2}$ around $\left(z_{k_{1}+10}, \cdots, z_{k 0}\right)$ in $\mathbb{R}^{k-k_{1}}$ so that, for any point $\left(z_{k_{1}+1}, \cdots, z_{k}\right)$ in $V_{2}$, there exists a unique point $\left(z_{1}, \cdots, z_{k_{1}}\right)$ in $V_{1}$ satisfying

$$
g\left(z_{1}, z_{2}, \cdots, z_{k}\right)=0
$$

There exists an open ball $B\left(t_{0}, \xi^{0}, u^{0}\right)$ around $\left(t_{0}, \xi^{0}, u^{0}\right)$ so that for any $(t, \xi, u)$ in $B\left(t_{0}, \xi^{0}, u^{0}\right)$,

$$
\begin{aligned}
& \left(Z_{k_{1}+1}(t, \xi, u), \cdots, Z_{k}(t, \xi, u)\right) \in V_{2} \\
& \left(Z_{1}(t, \xi, u), \cdots, Z_{k_{1}}(t, \xi, u)\right) \in V_{1}
\end{aligned}
$$

According to the assumption, we can assume $Z_{k_{1}+1}(t, \xi, u), \cdots, Z_{k}(t, \xi, u)$ are all observable in $B\left(t_{0}, \xi_{0}, u_{0}\right)$. Given two trajectories $\left(t, \xi^{1}(t), u^{1}(t)\right)$ and $\left(t, \xi^{2}(t), u^{2}(t)\right)$ in $B\left(t_{0}, \xi_{0}, u_{0}\right)$. If they have the same output, then for $i=k_{1}+1, \cdots, k$

$$
Z_{i}\left(t, \xi^{1}(t), u^{1}(t)\right)=Z_{i}\left(t, \xi^{2}(t), u^{2}(t)\right) .
$$

Because $z_{1}=Z_{1}(t, \xi, u), \cdots, z_{k_{1}}=Z_{k_{1}}(t, \xi, u)$ satisfy (2.4) and because the equation has a unique solution, we know that

$$
Z_{i}\left(t, \xi^{1}(t), u^{1}(t)\right)=Z_{i}\left(t, \xi^{2}(t), u^{2}(t)\right)
$$

for $i=1, \cdots, k_{1}$. So, $z_{1}, \cdots, z_{k_{1}}$ are $Z$-observable in $B\left(t_{0}, x i_{0}, u_{0}\right)$. Because the entire trajectory $(t, x(t), u(t))$ can be covered by finite many ball in which $z_{i}=Z_{i}(t, \xi, u), i=1, \cdots, k_{1}$, are $\mathrm{Z}$ observable, there exists an open neighborhood around the trajectory in which $z_{i}=Z_{i}(t, \xi, u), i=1, \cdots, k_{1}$, are $\mathrm{Z}$ observable. Therefore, $z_{1}=Z_{1}(t, \xi, u), \cdots, z_{k_{1}}=Z_{k_{1}}(t, \xi, u)$ are locally $Z$-observable in $U$.

\section{$3 \quad Z\left(T_{N}\right)$-observability}

Let us consider the following class of switched systems:

$$
\begin{aligned}
\dot{\xi} & =f_{q}(t, \xi, u), \quad q \in Q, \xi \in \mathbb{R}^{n}, u \in \mathbb{R}^{m} \\
Y & =h_{q}(t, \xi, u)
\end{aligned}
$$


Where $Q$ is a finite index set, $f_{q} \mathbb{R} \times \mathbb{R}^{n} \times \mathbb{R}^{m} \rightarrow \mathbb{R}^{m}$ is sufficiently smooth, the dwell time $\left[t_{i, 0}, t_{i, 1}\right]$ between two switches of the structure (i.e. change of $q$ ) is such that $\exists \tau_{\min }>0 /\left(t_{i, 1}-t_{i, 0}\right)>\tau_{\min }$ (this exclude Zeno phenomena). For the input $u$ in any time interval $\left[t_{i, 0}, t_{i, 1}\left[\subseteq\left[t_{i n i}, t_{e n d}[\right.\right.\right.$, we assume that $u(t)$ is bounded and $C^{\infty}$.

For switched systems the observation concept is strongly linked to the dwell time and the sequence of switch, thus it is important to recall (in our context) the following definition of hybrid time trajectory (Lygeros \& al., 2003) (see also (Goebel \& al., 2004)).

Definition 2 A hybrid time trajectory is a finite or infinite sequence of intervals $T_{N}=\left\{I_{i}\right\}_{i=0}^{N}$, such that

- $I_{i}=\left[t_{i, 0}, t_{i, 1}[\right.$, for all $0 \leq i<N$;

- For all $i<N t_{i, 1}=t_{i+1,0}$

- $t_{0,0}=t_{i n i}$ and $t_{N, 1}=t_{\text {end }}$

Moreover, we define $\left\langle T_{N}\right\rangle$ as the ordered list of $q$ associated to $T_{N}$ (i.e. $\left\{q_{0}, \ldots, q_{N}\right\}$ with $q_{i}$ the value of $q$ during the time interval $\left.I_{I}\right)$.

From these it is possible to define a new concept of observability:

DeFinition 3 The function $z=Z(t, \xi, u)$ is said to be $Z\left(T_{N}\right)$-observable in $U$ with respect to system 3.5 and hybrid time trajectory $T_{N}$ and $\left\langle T_{N}\right\rangle$ if for any two trajectories, $\left(t, \xi^{i}(t), u^{i}(t)\right), i=1,2$, in $U$ defined on the interval $\left[t_{\text {ini }}, t_{\text {end }}\right]$, the equality

$$
h\left(t, \xi^{1}(t), u^{1}(t)\right)=h\left(t, \xi^{2}(t), u^{2}(t)\right), \quad \text { a.e. in }\left[t_{\text {ini }}, t_{\text {end }}\right]
$$

implies

$$
Z\left(t, \xi^{1}(t), u^{1}(t)\right)=Z\left(t, \xi^{2}(t), u^{2}(t)\right), \text { a.e. in }\left[t_{i n i}, t_{\text {end }}\right]
$$

Suppose for any trajectory $(t, \xi(t), u(t))$ in $U$, there always exists an open set $U_{1} \subset U$ so that $(t, \xi(t), u(t))$ is contained in $U_{1}$ and $Z(t, x, u)$ is $Z\left(T_{N}\right)$ observable in $U_{1}$ with respect to system 3.5 and hybrid time trajectory $T_{N}$ and $\left\langle T_{N}\right\rangle$. Then, $z=Z(t, x, u)$ is said to be locally $Z\left(T_{N}\right)$-observable in $U$ with respect to system 3.5 and hybrid time trajectory $T_{N}$ and $\left\langle T_{N}\right\rangle$.

Lemma 5 Consider system 3.5 and hybrid time trajectory $T_{N}$ and $\left\langle T_{N}\right\rangle$. Let $U$ be an open set in time-state-control space. Suppose $Z(t, x(t), u(t))$ is always continuous under admissible control input. Suppose there exist $N+1$ linear projections $\left\{P_{i}\right\}$ so that 
(1) given any $0 \leq i \leq N, P_{i} Z(t, \xi, u)$ is $Z$-observable for $t \in\left[t_{i, 0}, t_{i, 1}[\right.$ and $(t, \xi(t), u(t)) \in U$;

(2) $\operatorname{Rank}\left\{P_{0}^{T}, \ldots, P_{N}^{T}\right\}=\operatorname{dim}(Z)=n_{z}$; and

(3) $\frac{d \bar{P}_{i} Z(t, \xi(t), u(t))}{d t}=0$ for $t \in\left[t_{i, 0}, t_{i, 1}[\right.$ and $(t, \xi(t), u(t)) \in U$, where $\left\{\bar{P}_{i}^{T}, P_{i}^{T}\right\}$ has full rank in $\mathbb{R}^{n_{z} \times n_{z}}$.

Then, $z=Z(t, \xi, u)$ is $Z\left(T_{N}\right)$-observable in $U$ with respect to system 3.5 and hybrid time trajectory $T_{N}$ and $\left\langle T_{N}\right\rangle$

Proof. By the assumption, within each $I_{i}$ the unobservable parts of the function $Z$ are constant; and they are observable in some other time intervals. More specifically, the integration of condition (3) yields

$$
\bar{P}_{i} Z(t, \xi(t), u(t))=\text { Constant }
$$

for $t$ in $I_{i}$. However, $P_{j} Z(t, \xi(t), u(t))$ is uniquely determined in the time interval $I_{j}$. Because $\operatorname{Rank}\left\{P_{0}^{T}, \ldots, P_{N}^{T}\right\}=n_{z}, \bar{P}_{i} Z$ is unquely determined. Because $\left\{\bar{P}_{i}^{T}, P_{i}^{T}\right\}$ has full rank, lemma 4 ensures that $Z(t, \xi(t), u(t))$ is observable in $I_{i}$ for all $0 \leq i \leq N$.

In the following, " $Z\left(T_{N}\right)$-observable in $U$ " means " $Z\left(T_{N}\right)$-observable in $U$ with respect to system 3.5 and hybrid time trajectory $T_{N}$ and $\left\langle T_{N}\right\rangle$ ".

REMARK 1 The Hybrid time trajectory $T_{N}$ and $\left\langle T_{N}\right\rangle$ influence the observability property in the way similar to an input. Therefore, in the future work it is interesting to define the universal hybrid time trajectory as the time trajectory which preserves the observability (see (Gauthier \& al., 2001) for the definition of universal input).

REMARK 2 Thus if system 3.5 is $Z\left(T_{N}\right)$ - observable, then it is possible to design an observer for the system. But this is beyond the scoop of the paper.

It is important to mention that all lemmas of the section 2 are valid for each time interval $I_{i}$. However, the generalization of the $Z\left(T_{N}\right)$ observability with nonlinear projections is not so obvious because, in this case, the projection can be function of unknown parameters. In the next section the usefulness of the $Z$-observability is highlighted by a system of networked oscillators. 


\section{Network Systems}

This section is dedicated to a networked system, more particularly to the influence of the small world effect with respect to the $Z$-observability. In (Watts \& al., 1998), D.S. Watts and S.H. Strogatz have given an example of small-world effect for a network with twenty vertices (see figure 1).
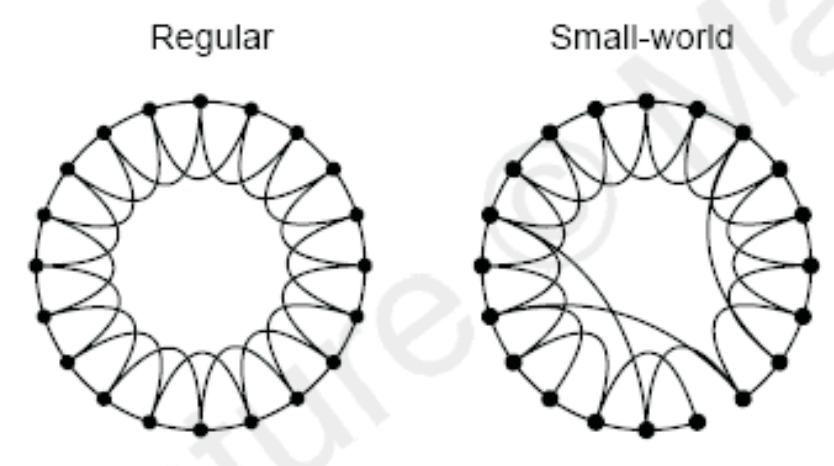

Figure 1: Networked of Watts and Strogatz

Hereafter, it is considered the same network where each vertex has the following dynamics:

$$
\begin{aligned}
& \dot{\xi}_{i, 1}=\xi_{i, 2}+\sum_{j \neq i, j=1}^{20} a_{i, j} \xi_{j, 1} \\
& \dot{\xi}_{i, 2}=-\xi_{i, 1}+m_{i}
\end{aligned}
$$

where $\xi_{i, 1}$ and $\xi_{i, 2}$ are the two variables state of the $i^{\text {th }}$ vertex. The dynamic at each vertex is similar. It is an oscillator coupled with four other ones. Moreover, only four $a_{i, j}=0.25$ all other one are equal to zero. And, $m_{i}$ is the fault. Only some variables $\xi_{i, 1}$ are measurable.

First of all let us consider the following regular network (i.e. $a_{i, i-2}=a_{i, i-1}=$ $a_{i, i+1}=a_{i, i+2}=0.25$ all other $a_{i, j}=0$, where $a_{i, 21}=a_{i, 1}, a_{i, 22}=a_{i, 2}$, $a_{i, 0}=a_{i, 19}$ and $\left.a_{i,-1}=a_{i, 18}\right)$. Consider the case of no fault in the system. With one output, the system is not observable, and the dimension of the observable space is only 20; with two successive outputs the dimension of the observable space is 36 ; with three successive outputs the dimension of the observable space is 38 ; and only for four and more successive outputs the regular system is observable. Moreover, if a fault detection problem is considered vertex by vertex, then the state of a vertex can be observable 
even if the entire system is not. Define $Z(t, \xi, m)$ for the $i^{t h}$ vertex as follows

$$
Z(t, \xi, m)=\left(\xi_{i, 2}, m_{i}\right)^{T}
$$

The outputs are defined by $\xi_{i-2,1}=y_{-2}, \xi_{i-1,1}=y_{-1}, \xi_{i, 1}=y_{0}, \xi_{i+1,1}=y_{1}$ and $\xi_{i+2,1}=y_{2}$ the following differential algebraic equations are obtains:

$$
\begin{aligned}
& D y_{0}=\xi_{i, 2}+0.25\left(y_{-2}+y_{-1}+y_{1}+y_{2}\right) \\
& D^{2} y_{0}=-y_{0}+m_{i}+0.25\left(D y_{-2}+D y_{-1}+D y_{1}+D y_{2}\right)
\end{aligned}
$$

Then the lemma 1 guaranty that $Z(t, \xi, m)=\left(\xi_{i, 2}, m_{i}\right)^{T}$ is $Z$-observable. In this case, the entire system is not observable without information about all $m_{i}$. However, the fault of a vertix is observable

Now consider the same network (with the same vertex dynamics) but with small world effect (see figure 1). Assuming no fault, then with one output the dimension of the observable space is 36 ; with two successive outputs the system is observable. This seems a beneficial effect on observability of small world network architecture (i.e. less outputs are necessary in order to observe the full state of the system). Nevertheless, from this architecture and without assumption on the $m_{i}$, it is impossible to detect fault on each vertex with only the knowledge of the two upper and lower outputs neighborhood (i.e. $\xi_{i-2,1}=y_{-2}, \xi_{i-1,1}=y_{-1}, \xi_{i, 1}=y_{0}, \xi_{1+1,1}=y_{1}$ and $\xi_{i+2,1}=y_{2}$ ). This shows that the sensors repartition is of first importance for fault detection in network with small world structure. Moreover the choice of sensor localization must be done with the Z-observable concept because detection of $m_{i}$ is requested and the observation of the full state is not necessary.

\section{Switched System}

Let us consider the following switched system without jump.

$$
\begin{aligned}
& \dot{x}_{1}=-x_{1}+q_{i, 1} x_{2}+q_{i, 2} x_{3} \\
& \dot{x}_{2}=-q_{i, 1}\left(q_{i, 1} x_{2}+q_{i, 2} x_{3}\right) \\
& \dot{x}_{3}=-q_{i, 2}\left(q_{i, 1} x_{2}+q_{i, 2} x_{3}\right)
\end{aligned}
$$

where $y=x_{1}$ is the output.

The hybrid time trajectory $T_{N}$ is the set of following time interval $\left[i \tau,(i+1) \tau\left[_{i=0}^{\infty}\right.\right.$ with $\tau=\tau_{\min }>0$ and $\left\langle T_{N}>\right.$ the order list of $q_{i}=\left(q_{i, 1}, q_{i, 2}\right)$ is defined as 
follow:

$$
\begin{aligned}
& q_{1}=(1,-1) \quad \text { and for } j=3 k+1, q_{j}=q_{1} \\
& q_{2}=(-1,1) \quad \text { and for } j=3 k+2, q_{j}=q_{2} \\
& q_{3}=(1,1) \quad \text { and for } j=3 k, q_{j}=q_{3}
\end{aligned}
$$

For a function $z=Z(t, x)=\left(x_{1}(t), x_{2}(t), x_{3}(t)\right)^{T}$, a choice of projections matrixes is:

$$
\begin{aligned}
& P_{1}=P_{2}=\left(\begin{array}{ccc}
1 & 0 & 0 \\
0 & 1 & -1
\end{array}\right) \\
& \bar{P}_{1}=\bar{P}_{2}=\left(\begin{array}{lll}
0 & 1 & 1
\end{array}\right)
\end{aligned}
$$

and

$$
P_{3}=\left(\begin{array}{ccc}
1 & 0 & 0 \\
0 & 1 & 1
\end{array}\right), \quad \bar{P}_{3}=\left(\begin{array}{lll}
0 & 1 & -1
\end{array}\right)
$$

Moreover, the $P_{j}$ and $\bar{P}_{j}$ for $j>3$ are defined as follow:

$$
\begin{array}{lll}
P_{j}=P_{1} & \text { and } \bar{P}_{j}=\bar{P}_{1} & \text { for } j=3 k+1 \\
P_{j}=P_{2} & \text { and } \bar{P}_{j}=\bar{P}_{2} & \text { for } j=3 k+2 \\
P_{j}=P_{3} & \text { and } \bar{P}_{j}=\bar{P}_{3} & \text { for } j=3 k
\end{array}
$$

From the lemma 5 it is clear that all state components are $Z\left(T_{N}\right)$ observable (if the hybrid time trajectory ends at least after $3 \tau$ ), even if the function $z$ is not observable for any couple $\left(T_{i}, q_{i}\right)$.

\section{References}

Angeli, S. and E. Sontag, Forward completeness, unboundedness observability and their Lyapunov characterisations, Systems 83 Control Lett., Vol 38: pp 209217, 1999.

Bemporad, A., G. Ferrari-Trecate, and M. Morari. Observability and Controllability of Piecewise Affine and Hybrid Systems. IEEE Trans on Automatic Control, 45 No 10, pp. 1864-1876., 2000

Boutat, D., A. Benali and J.P. Barbot. (2004). About the observability of piecewise dynamical systems. NOLCOS-2004. (CD-ROM).

Diop, S. and Fliess, S. On nonlinear observability. In C. Commault and coll., editors, Proceeding of ECC'91, Volume 1, pp 152-157. Hermès, 1991. 
Gauthier J-P, I. A. K. Kupka, Deterministic Observation Theory \& Applications, Cambridge University Press, 2001.

R. Goebel J. Hespanha, A R. Teel, C. Cai and R. Sanfelice, Hybrid systems: generalized solutions and robust stability, IFAC, Nolcos 04 .

Gong, Q, I. M. Ross, W. Kang, A Pseudospectral Observer for Nonlinear Systems, Discrete and Continuous Dynamical Systems, Series B, to appear

Kang, W., Moving Horizon Numerical Observers of Nonlinear Control Systems, IEEE Trans. on Automat. Contr., Vol. 51, No. 2, pp. 344-350, 2006.

Krener A. J. and W. Kang, Locally Convergent Nonlinear Observers, SIAM J. on Control and Optimization, Vol. 42, No. 1, pp. 155-177, 2003.

Hermann, R. and Krener, A.J., Nonlinear controllability and Observability, IEEE TAC, 22, No 9, pp. 728-740,1977

Levant A., (1998), Robust Exact Differentiation via sliding mode terchnique, In Automatica, Vol. 34, No. 3, pp. 379-384, 1998

Lygeros, J., H.K. Johansson, S. N. Simć, J. Zhang and S.S. Sastry, Dynamical Properies of Hubrid Automata, in IEEE Trans. on Autom. Control, Vol. (48), No. 1, pp. 2-17, January, 2003.

Nijmeijer, H. and M.Y Mareels, An Observer looks at synchronization, IEEE-TCS I Fund. Theory Appl. 44, pp. 882-890, 1997

$\mathrm{Vu}, \mathrm{L}$. and D. Liberzon, Invertibilty of switched linear systems. IEEE 45th CDC, San Diego 2006

Watts, D.S. and S.H. Strogatz, Collective dynamics of 'small-world' networks.Nature, Vol 393, pp 440-443, 4 June 1998. 